\title{
Manifestaciones reumatológicas y sistémicas en pacientes con enfermedad inflamatoria intestinal
}

\author{
F.A. Sommerfleck ${ }^{1}$, E.E. Schneeberger ${ }^{1}$, M.A. Suarez Lissi', F. Chiardola ${ }^{1}$, A. Sambuelli ${ }^{2}$, O. Marconi ${ }^{2}$, \\ M.G. Rosemffet ${ }^{1,}$ J.A. Maldonado Cocco ${ }^{1}$, G. Citera ${ }^{1}$ \\ ${ }^{1}$ Sección Reumatología, Instituto de Rehabilitación Psicofísica. \\ ${ }^{2}$ Sección Enfermedades Inflamatorias Intestinales, Hospital Dr. B. Udaondo. Buenos Aires.
}

\section{Resumen}

Introducción: La Enfermedad Inflamatoria Intestinal (Ell) comprende la Enfermedad de Crohn (EC) y la Colitis Ulcerosa (CU). La frecuencia del compromiso sistémico en la Ell es entre 20 y $40 \%$, y las manifestaciones más frecuentes son las dermatológicas, oculares, articulares y hepatobiliares. Ante la falta de datos sobre la frecuencia y características de dichas manifestaciones en nuestra población, decidimos evaluar la prevalencia de las manifestaciones clínicas extraintestinales en pacientes argentinos con diagnóstico de Ell, evaluar sus características, determinar su asociación con la CU y/o EC y examinar el compromiso axial radiológico de estos pacientes.

Materiales y métodos: Se estudiaron 95 pacientes consecutivos con diagnóstico establecido de Ell. Se completó un cuestionario registrando datos demográficos y clínicos de la Ell. Dos médicos realizaron un examen físico completo reumatológico. Se realizaron radiografías de columna lumbar y cervical, pelvis panorámica y sacroilíacas por técnica de Fergusson puntuadas según criterios de New York. Las radiografías fueron leídas por un investigador independiente que desconocía los datos clínicos de los pacientes.

Resultados: Se incluyeron 95 pacientes, 39,9\% eran de sexo masculino. La edad mediana fue de 37 años y la mediana de tiempo de evolución de la Ell fue de 6 años. En relación al diagnóstico, 75,8\% tenían CU y 22,11\% EC; un paciente presentaba ambos diagnósti$\cos (\mathrm{CU}$ y EC) y otro tenía enfermedad intestinal indiferenciada. Las manifestaciones no reumatológicas más frecuentes fueron: úlceras orales $38,9 \%$, uveítis $10,5 \%$, cervicitis/uretritis $8,4 \%$, eritema nodoso $6,3 \%$, psoriasis $2,1 \%$ y pioderma gangrenoso $1,1 \%$. En cuanto a las manifestaciones reumatológicas: lumbalgia inflamatoria $44,2 \%$, artritis periférica $27,4 \%$, talalgia $24,2 \%$, dactilitis $13,7 \%$ y entesitis $11,6 \%$. No se observaron diferencias significativas en la frecuencia de estas manifestaciones entre los distintos tipos de Ell. Sacroileítis con graduación mayor o igual a 2 se detectó en $23,8 \%$ de los pacientes, y ésta fue significativamente más frecuente en los pacientes con EC. La sacroileítis radiológica estuvo presente en 16 pacientes siendo asintomática en un $62,5 \%$ y en todos los casos fue asimétrica.

Discusión: El análisis de nuestros datos confirma las características reportadas en otras series y creemos importante el poder tener datos propios sobre los pacientes con enfermedad intestinal inflamatoria.

Palabras clave: enfermedad inflamatoria intestinal, espondiloartritis, colitis ulcerosa, enfermedad de Crohn.

\section{Abstract}

Introduction: Inflammatory Bowel Disease (IBD) includes Crohn's Disease (CD) and Ulcerative Colitis (UC). The frequency of systemic involvement in IBD is between 20 and $40 \%$, and most common manifestations are dermatologic, ocular, articular and hepatobiliary. In the absence of data on the frequency and characteristics of such events in our population, we decided to evaluate the prevalence of extraintestinal manifestations in argentinian patients diagnosed with IBD, evaluate their characteristics, determine its association with UC and/ or CD and examine the radiographic engagement of these patients. Materials and Methods: 95 consecutive patients with an established diagnosis of IBD were included. A questionnaire was completed collecting demographic and clinical data of IBD patients. Two physicians performed a complete rheumatological examination. Radiographs were read by an independent investigator blinded to the clinical data of patients.

Results: 95 patients were included, $39.9 \%$ were male. The median age was 37 years and median duration of IBD was 6 years. $75.8 \%$ had UC and $22.11 \% \mathrm{CD}$, one patient had both diagnoses (UC and CD) and one had undifferentiated intestinal disease. The most frequent non-rheumatological manifestations were $38.9 \%$ oral ulcers, uveitis $10.5 \%$, cervicitis/urethritis $8.4 \%$, erythema nodosum $6.3 \%$, psoriasis $2.1 \%$ and pyoderma gangrenosum $1.1 \%$. As for rheumatological manifestations: inflammatory back pain $44.2 \%$, peripheral arthritis $27.4 \%$, heel pain $24.2 \%$, dactylitis $13.7 \%$ and enthesitis $11.6 \%$. No significant differences in the frequency of these manifestations between different types of IBD were observed. Sacroiliitis with greater than or equal to 2 graduation was detected in $23.8 \%$ of patients, and this was significantly more frequent in patients with CD. Radiological sacroiliitis was present in 16 patients remain asymptomatic in $62.5 \%$ and in all cases was asymmetric.

Discussion: The analysis of our data confirms the characteristics reported in other series. We believe it is important to have data of our patients with inflammatory bowel disease.

Key words: inflamatory bowel disease, spondyloarthritis, ulcerative colitis, Crohn's disease. 


\section{Introducción}

La Enfermedad Inflamatoria Intestinal (EII) es una enfermedad con compromiso del tracto gastrointestinal y consiste en dos entidades diferentes: la Enfermedad de Crohn (EC) y la Colitis Ulcerosa (CU)1. La CU se caracteriza por episodios recurrentes de inflamación limitada a la mucosa intestinal colónica comprometiendo principalmente el recto, mientras que la EC puede comprometer cualquier segmento del tracto digestivo y presenta compromiso típicamente trasmural y con frecuencia se asocia a fibrosis y a obstrucción intestinal.

Según reportes, la EII presenta compromiso sistémico entre 20 y $40 \%$ y las manifestaciones más frecuentes son las dermatológicas (piel y mucosas), oculares, articulares y hepatobiliares. ${ }^{2,3}$ Las manifestaciones reumatológicas más frecuentes son artritis periférica y axial y también entesitis y osteopatía hipertrófica ${ }^{4}$. La artritis periférica se presenta con una frecuencia del 5 al 30\%, es oligoarticular, asimétrica a predominio de miembros inferiores y su distribución es igual en ambos sexos. La aparición de la artritis suele ser simultánea o posterior a la EII, y los brotes articulares e intestinales suelen coincidir. Usualmente esta artritis es autolimitada, no erosiva y suele ser más frecuente en la EC 5,6. En relación al compromiso axial, la lumbalgia inflamatoria se ha reportado con frecuencias cercanas al 30\%. El compromiso axial puede presentarse en 2 formas: a) Espondilitis Anquilosante (EA) clínica y radiológicamente similar a la EA pura y b) Sacroileítis aislada. Según los distintos reportes, la EA se presenta con una frecuencia variable del 1 al $25 \%$. A diferencia de la EA pura, la frecuencia según género es semejante y el compromiso axial suele ser menos severo con menor limitación de la movilidad espinal ${ }^{7-9}$. El comienzo de la espondilitis es independiente del comienzo de la EII pudiendo precederla en años y su desarrollo está relacionado con la presencia de HLA B27 y no está vinculado a la enfermedad intestina ${ }^{10}$. La sacroileítis (SI) aislada se observa con una frecuencia que varía entre el 2 y $25 \%$. Existen reportes controvertidos en relación a las características del compromiso de las SI, clásicamente se ha descripto como simétrica y progresiva aunque estudios ulteriores observaron una alta frecuencia de compromiso unilateral. Las entesopatías se desarrollan en un 6 a un 15\% y son más comunes a nivel del tendón de Aquiles y la fascia plantar ${ }^{11,12}$.

En nuestro país, no conocemos la frecuencia y características de las manifestaciones extraintestinales, por eso el objetivo de este trabajo fue determinar la prevalencia de las manifestaciones clínicas en pacientes con diagnóstico de EII, evaluar sus características, determinar su asociación con la CU y/o la EC y examinar el compromiso radiológico de estos pacientes.

\section{Materiales y métodos}

Se estudiaron pacientes mayores de 18 años de edad consecutivos desde enero a noviembre del 2002, con diagnóstico establecido de EII atendidos en la sección de "Enfermedades inflamatorias intestinales" del Hospital Udaondo (Centro gastroenterológico de referencia). Se consignaron datos demográficos (edad, sexo) y clínicos (tiempo de evolución de la enfermedad, antecedentes familiares, tipo de EII y manifestaciones clínicas extraintestinales). Dos médicos realizaron un examen físico reumatológico completo. Se incluyó la evaluación clinimétrica de los pacientes registrando medición de la flexión lumbar por prueba de Schober, expansión torácica, distancia occipucio-pared y dedos-piso, así como la presencia de articulaciones inflamadas mediante el recuento articular de 44 . Se realizaron radiografías de pelvis, de articulaciones sacroilíacas por técnica de Fergusson, de columna cervical, dorsolumbar en posición de frente, perfil y pelvis panorámica. Las radiografías fueron leídas por un investigador independiente que desconocía los datos clínicos de los pacientes. En columna se evaluó la presencia de sindesmofitos, cuadratura vertebral y discitis. En pelvis se valoró compromiso de la articulación coxofemoral y de entesitis. El compromiso de las articulaciones sacroilíacas se puntuó según criterios de New York ${ }^{13}$.

Para el análisis estadístico se determinó la estadística descriptiva expresándose los datos continuos como medianas y rango intercuartilo (RIC) y los datos categóricos como frecuencias. Las comparaciones entre datos categóricos se realizó por test de Chi cuadrado o test exacto de Fisher según correspondiera y los datos continuos por test de Mann-Whitney. Se establecieron correlaciones entre distintas variables por el test no paramétrico de Spearman y en el caso de las correlaciones múltiples el valor de $\mathrm{p}$ fue corregido por el número de comparaciones. Un valor de $\mathrm{p}<0,05$ fue considerado significativo.

\section{Resultados}

Se incluyeron 95 pacientes, 58 (61,1\%) de sexo femenino. La edad mediana fue 37 años (RIC 25-54) y la mediana de tiempo de evolución de la EII fue de 6 años (RIC 3-11). En relación al diagnóstico, 72 pacientes $(75,8 \%)$ tenían CU y $21(22,11 \%)$ EC; un paciente tuvo asociación de CU y EC y otro enfermedad inflamatoria intestinal indiferenciada.

Las manifestaciones extraintestinales más frecuentes fueron: úlceras orales $(38,9 \%)$, uveítis $(10,5 \%)$, cervicitis/ uretritis $(8,4 \%)$, eritema nodoso $(6,3 \%)$, psoriasis $(2,1 \%)$, pioderma gangrenoso $(1,1 \%)$. No se observaron diferencias en la frecuencia de estas manifestaciones entre los distintos tipos de EII (Tabla 1). 


\begin{tabular}{|l|c|c|c|}
\hline Manifestación & CU $\mathbf{n = 7 2} \mathbf{n}(\%)$ & EC $\mathbf{n = 2 1} \mathbf{n}(\%)$ & $\mathbf{p}$ \\
\hline Úlceras orales & $27(37,5)$ & $9(42,9)$ & NS \\
\hline Uveítis & $8(11,1)$ & $2(9,5)$ & NS \\
\hline Cervicitis / Uretritis & $6(8,3)$ & $1(4,8)$ & NS \\
\hline Eritema nodoso & $4(5,6)$ & $2(9,5)$ & NS \\
\hline Psoriasis & 0 & $1(4,8)$ & NS \\
\hline Pioderma gangrenoso & $1(1,4)$ & 0 & NS \\
\hline
\end{tabular}

Tabla 1. Manifestaciones extraintestinales no reumatológicas en 93 pacientes con enfermedad inflamatoria intestinal.

Las principales manifestaciones reumatológicas fueron: lumbalgia inflamatoria $(44,2 \%)$, artritis periférica $(27,4 \%)$, talalgia $(24,2 \%)$, dactilitis $(13,7 \%)$ y entesitis $(11,6 \%)$. Tampoco se observaron diferencias significativas de las frecuencias de estas manifestaciones entre los distintos tipos de EII (Tabla 2).

En cuanto al examen físico reumatológico, los principales hallazgos observados fueron: maniobras para sacroileítis positiva $(21,1 \%)$, entesitis aquiliana $(3,2 \%)$ y artritis periférica $(2,1 \%)$.

Se realizaron radiografías en 65 pacientes; las principales características radiológicas observadas fueron sacroileítis grado mayor o igual a 2 en un $23,8 \%$ de los pacientes, y ésta fue significativamente más frecuente en los pacientes con EC en relación a los pacientes con CU, $50 \%$ vs. $18,9 \%$ respectivamente $(p=0,04)$. Pinzamiento coxofemoral se observó en $4,76 \%$ de los pacientes, periostitis y proliferaciones óseas irregulares o "whiskering" en 6,34\%, cuadratura vertebral dorsolumbar en $9,5 \%$ y sindesmofitos en un solo paciente a nivel cervical que padecía de EC.

La sacroileítis radiológica con grados mayores o iguales a dos según criterios de New York, estuvo presente en 16 pacientes, siendo un $62,5 \%$ de los casos asintomática y en todos los casos, asimétrica. La sacroileítis radiológica fue significativamente más frecuente en los pacientes con historia familiar de psoriasis que en los que no tenían antecedentes (57,1\% vs. $19,6 \%$ respectivamente, $\mathrm{p}=0,04)$. La presencia de lumbalgia correlacionó discretamente con la talalgia $(r=0,20$, $\mathrm{p}=0,04)$. La presencia de sacroileítis se correlacionó con el tiempo de evolución de la enfermedad, $(r=0,28, p=0,03)$.

Solo un paciente fue diagnosticado como Espondilitis Anquilosante (EA) según criterios de New York modificados $1987^{13}$.

\begin{tabular}{|l|c|c|c|}
\hline $\begin{array}{l}\text { Manifestaciones } \\
\text { reumatológicas }\end{array}$ & CU $\mathbf{n = 7 2} \mathbf{n}(\%)$ & EC $\mathbf{n}=\mathbf{2 1} \mathbf{n}(\%)$ & $\mathbf{p}$ \\
\hline Lumbalgia inflamatoria & $32(44,4)$ & $8(38,1)$ & NS \\
\hline Artritis periférica & $19(26,4)$ & $6(28,6)$ & NS \\
\hline Talalgia & $16(22,2)$ & $7(33,3)$ & NS \\
\hline Dactilitis & $8(11,1)$ & $4(19)$ & NS \\
\hline Entesitis & $9(12,5)$ & $2(9,5)$ & NS \\
\hline
\end{tabular}

Tabla 2. Manifestaciones reumatológicas en 93 pacientes con enfermedad inflamatoria intestinal.

\section{Discusión}

Los resultados de nuestro estudio revelan que existe un elevado porcentaje de pacientes con enfermedad intestinal inflamatoria que a lo largo de su evolución sufren una o más manifestaciones extraintestinales. Siendo la frecuencia de las mismas similar a los reportes de la literatura ${ }^{14,15}$.

En la EII una de las manifestaciones extraintestinales más frecuentes son las manifestaciones reumatológicas. La artritis periférica tiene importante variabilidad según diferentes reportes entre $9 \%$ y $53 \%$, siendo en nuestra serie de $27,4 \% \%^{16-18}$.

En relación al compromiso axial, a diferencia de los pacientes con EA, la sacroileítis en este grupo de pacientes suele ser asintomática y menos discapacitante ${ }^{19}$. Dekker-Saeys y cols., en 1978, reportaron que un $10 \%$ de una cohorte de pacientes con EII presentaban sacroileítis radiológica, en ninguna de ellas había compromiso lumbar radiológico y en $91 \%$ de los casos fue asintomática ${ }^{20}$. Cuando realizamos, en el 2011, un análisis de los pacientes de la base RESPON$D^{2} A^{21}$ para describir las diferencias entre las características de la Espondilitis asociada a psoriasis y asociada a la EII con la Espondilitis Anquilosante, se observó que los pacientes con artritis psoriática y EII tenían menor sintomatología axial y menor compromiso radiológico que los pacientes con EA pura. Acorde a dichos datos, en este trabajo, hemos visto que la sacroileítis fue en su mayoría asintomática (62,5\%).

Éste es el primer estudio argentino en evaluar las manifestaciones reumatológicas en los pacientes con EII, pero sin embargo tiene ciertas limitaciones. Primero, es un estudio de corte transversal por lo cual muchos de los datos fueron recabados en forma retrospectiva. Segundo, los pacientes no fueron evaluados según las nuevas recomendaciones ASAS $^{22}$ ya que este estudio fue realizado en el 2002,

\begin{tabular}{|c|c|c|c|c|c|c|c|}
\hline \multicolumn{8}{|c|}{ Lumbalgia } \\
\hline \multicolumn{4}{|c|}{$\mathrm{SI}(\mathrm{n}=42)$} & \multicolumn{4}{|c|}{ NO $(n=53)$} \\
\hline \multicolumn{2}{|c|}{$\operatorname{MSI}(+) n=18$} & \multicolumn{2}{|c|}{$\operatorname{MSI}(-) n=24$} & \multicolumn{2}{|c|}{ MSI (+) n=2 } & \multicolumn{2}{|c|}{ MSI (-) n=51 } \\
\hline SI Rx & $\mathrm{Rx} N$ & SI Rx & Rx N & SI Rx & Rx N & SI Rx & Rx N \\
\hline $2 / 15(13,3 \%)$ & $13 / 15(86,7 \%)$ & $4 / 13(30,8 \%)$ & $9 / 13(69,2 \%)$ & $0 / 1$ & $1 / 1(100 \%)$ & $10 / 36(27,8 \%)$ & $26 / 36(72,2 \%)$ \\
\hline
\end{tabular}

Tabla 3. Evaluación clínica y radiológica de los pacientes con Ell. 
y tercero, las radiografías fueron evaluadas utilizando solamente los criterios de New York para sacroileítis y otros índices radiológicos no fueron utilizados. Además solamente se diagnosticó un paciente como EA. Esto también puede deberse a que en esa época la demora al diagnostico era mayor porque los pacientes eran solo clasificados como Espondilitis Anquilosante cuando cumplían los criterios de New York. Es probable que actualmente si utilizáramos los criterios ASAS 2009, el número de casos de SpA axial no radiológica aumentaría. Recientemente, en un estudio realizado en la Mayo $\mathrm{Clinic}^{23}$, se analizó una cohorte de 351 pacientes con Colitis Ulcerosa en la cual solo 1 paciente fue diagnosticado como EA según criterios de New York mientras que 40 pacientes presentaban SpA axial según criterios ASAS 2009, esto refuerza nuestra suposición.

En resumen, el análisis de nuestros datos confirma las características reportadas en otras series y creemos importante el tener datos en Argentina sobre pacientes con enfermedad inflamatoria intestinal y su compromiso extraintestinal. Se requieren más estudios a fin de poder conocer mejor a nuestra población de pacientes con enfermedad intestinal inflamatoria.

\section{Bibliografía}

1. Larsen S, Bendtzen K, Nielsen OH. Extraintestinal manifestations of inflammatory bowel disease: epidemiology, diagnosis, and management. Ann Med 2010; 42(2):97-114.

2. Greenstein AJ, Janowitz HD, Sachar DB. The extra-intestinal complications of Crohn's disease and ulcerative colitis: a study of 700 patients. Medicine (Baltimore) 1976; 55:401-12.

3. Loftus EV Jr. Clinical epidemiology of inflammatory bowel disease: Incidence, prevalence, and environmental influences. Gastroenterology 2004; 126(6):1504-17.

4. Orchard TR, Wordsworth BP, Jewell DP. Peripheral arthropathies in inflammatory bowel disease: their articular distribution and natural history. Gut 1998; 42(3):387-91.

5. D'Incà R, Podswiadek M, Ferronato A. Articular manifestations in inflammatory bowel disease patients: a prospective study. Dig Liver Dis 2009; 41(8):565-9.

6. Mauro-Filho Gde L, Ribeiro S. Articular manifestations of inflammatory bowel disease. Arq Gastroenterol 1985; 22(4):186-91.

7. van der Linden S, van der Heijde D. Ankylosing spondylitis. Clinical features. Rheum Dis Clin North Am 1998; 24:663-73.

8. Stolwijk C, Pierik M, Landewé R. Prevalence of self-reported spondyloarthritis features in a cohort of patients with inflammatory bowel disease. Can J Gastroenterol 2013; 27(4):199-205.

9. Shivashankar R, Loftus EV Jr, Tremaine WJ. Incidence of spondyloarthropathy in patients with Crohn's disease: a population-based study. J Rheumatol 2012; 39(11):2148-52.
10. González-Roces S, Alvarez V. HLA-B27 structure, function, and disease association. Curr Opin Rheumatol 1996; 8(4):296-308.

11. Wright V, Watkinson G. Sacro-ilitis and ulcerative colitis. Br Med J 1965; 2:675-80.

12. Bernstein CN, Wajda A, Blanchard JF. The clustering of other chronic inflammatory diseases in inflammatory bowel disease: a population-based study. Gastroenterology 2005; 129:827-36.

13. van der Linden S, Valkenburg HA, Cats A. Evaluation of diagnostic criteria for ankylosing spondylitis. A proposal for modification of the New York criteria. Arthritis Rheum 1984; 27(4):361-8.

14. Stebbings S, Jenks K, Treharne GJ, García JA. Validation of the Dudley Inflammatory Bowel Symptom Questionnaire for the assessment of bowel symptoms in axial SpA: prevalence of clinically relevant bowel symptoms and association with disease activity. Rheumatology (Oxford) 2012 May; 51(5):858-65.

15. Vounotrypidis P, Efremidou E, Zezos P. Prevalence of joint hypermobility and patterns of articular manifestations in patients with inflammatory bowel disease. Gastroenterol Res Pract 2009; 924-38.

16. Maeda K, Okada M, Yao T, Sakarai T, Iida M, Fuchigami T. Intestinal and extraintestinal complications of Crohn disease predictors and cumulative probability of complications. J Gastroentetrol 1994; 29:577-82.

17. Acheson Ed. An association between ulcerative colitis, regional enteritis, and ankylosing spondylitis. Q J Med 1960; 29:489-99.

18. Haslock I. Arthritis and Crohns disease. A family study. Ann Rheum Dis 1973; 32:479-486.

19. Voulgari PV. Rheumatological manifestations in inflammatory bowel disease. Ann Gastroenterol 2011; 24:173-80.

20. Dekker-Sayes BJ, Meuwissen SGM, Tytgat GNJ. Prevalence of pheripheral arthritis, sacroileitis and ankylosing spondylitis in patient suffering from inflammatory bowel disease. Ann Rheum Dis 1978; 37:33-5.

21. Pérez Alamino R, Maldonado Cocco JA, Citera G, et al. Differential features between primary ankylosing spondylitis and spondylitis associated with psoriasis and inflammatory bowel disease. J Rheumatol 2011; 38(8):1656-60.

22. Rudwaleit M, Landewé $R$, van der Heijde $D$, et al. The development of Assessment of SpondyloArthritis international Society classification criteria for axial spondyloarthritis (part I): classification of patients by expert opinion including uncertainty appraisal. Ann Rheum Dis 2009; 68(6):770-6.

23. Shivashankar R, Loftus EV, Tremaine WJ, et al. Incidence of Spondyloarthropathy in Patients with Ulcerative Colitis: A Population-based Study. J Rheumatol 2013; 40:1153-7. 